\title{
Research on the Resource Utilization of the Carrier Aircraft Security Phase
}

\author{
Liang Ma \\ Department of Surface Ship Command, Dalian Naval Academy, Dalian, 116018, China \\ 446982051@qq.com
}

Keywords: Guarantee resource utilization; Carrier guarantee

\begin{abstract}
To improve the efficiency of carrier aircraft security as the goal, around its problems existing in the fusion of resource using, equipment configuration, comb, and analyzes on the aircraft carrier aircraft security resources utilization, the problems existing in the equipment configuration as the breakthrough point, study problems of ship machine integration resources to plan as a whole, fully absorb the latest foreign ideas, use scientific theory research method was optimized, in its integration of resource using, equipment configuration and so on optimization and improvement measures are put forward Suggestions, exploring the aircraft to ensure maximum efficiency, and provide reference for subsequent carrier construction Suggestions. Research results can provide ship machine integration, equipment configuration of resource using the optimized solution and the suggestion, for subsequent carrier construction development provide important reference and guidance for the basis, to promote the construction of aircraft carrier fighting capacity has certain reference significance and reference value, military benefit is remarkable.
\end{abstract}

\section{Introduction}

The suddenness, complexity, variability and efficiency of the modern war, require the aircraft can be prepared to fight, anytime and anywhere in the complex battlefield environment to ensure fast aircraft, aircraft, more good. So, efficient security operation is not only ensure the normal use of its functions, the basis of the relationship with the entire aircraft forces the quick reaction capability, out strength and sustained high-intensity combat ability such as the key link, to a certain extent determines the outcome of the battle. Therefore, the basic and prerequisite for the formation of operational capability of the aircraft carrier is that the carrier guarantee is strong and efficient. Reasonable resource utilization and equipment allocation will improve the carrier's operational capability, and it will also be an inevitable choice to get out of a less expensive and more efficient equipment guarantee. According to the requirements of the carrier operations, reasonably allocate space within the limited security resources, coordination and planning equipment, management of each aircraft security work progress, and the safeguard work order, to avoid unreasonable impact protection efficiency of resource using.

\section{Operational Analysis of Operation Safety}

Flight security basic process is: after the orders from a superior, crew and safeguard equipment in place quickly, and to pull the aircraft to the designated place, in order to carry out the four basic preparation: aircraft refueling, aircraft air oxygen filling, mounted weapons, at the same time the maintenance checks. After the preparation, the mechanic will review the work and wait for the pilot to inspect the aircraft. The pilot will be ready to take off after he has passed the test, and the machine will be ready for work.

The actual preparation is very complex integrated system. its operation, involves the guarantee of equipment type and quantity and various professionals are many, in order to facilitate research and understanding, based on the principles of concentration of the complex security operation by clustering processing will each professional security work content reduced to refuel, oxygen filling, charging, play, and other related inspection work. The work can not be carried out at the same time, and the work must be finished at the end and the operation time is the longest. Set up the air 
conditioning station, the machine hydraulic station, the engine water supply station, the compressed air station, the nitrogen station and other related inspection operations as parallel machines. Schematic diagram 1 shows:

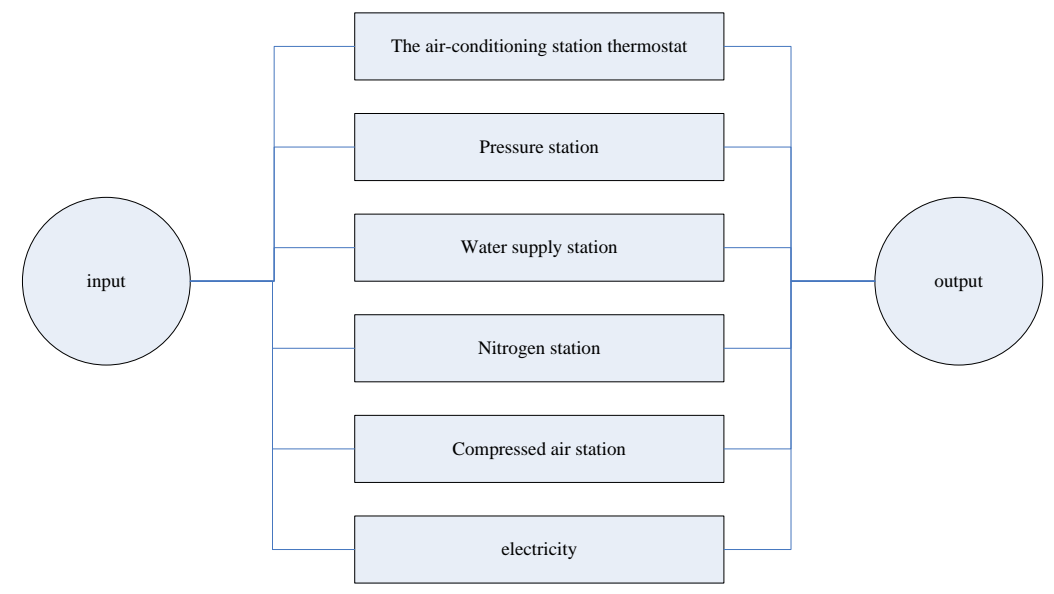

Figure 1. The parallel machine composition diagram

Because of the refueling, the power to charge, the oxygen and the bomb can't work at the same time, and the bomb must be done in other jobs. Therefore, the maintenance time of the machine is mainly composed of refueling, electricity, oxygenation and hanging cartridge.

Therefore, the schematic diagram of the aircraft security of a single plane can be shown as figure 2. With three basic maintenance preparation not conducted on a plane at the same time, and no correlation between each work, namely the three stages of the work order is not strictly limited, it's for machine maintenance guarantee to provide the premise for process optimization.

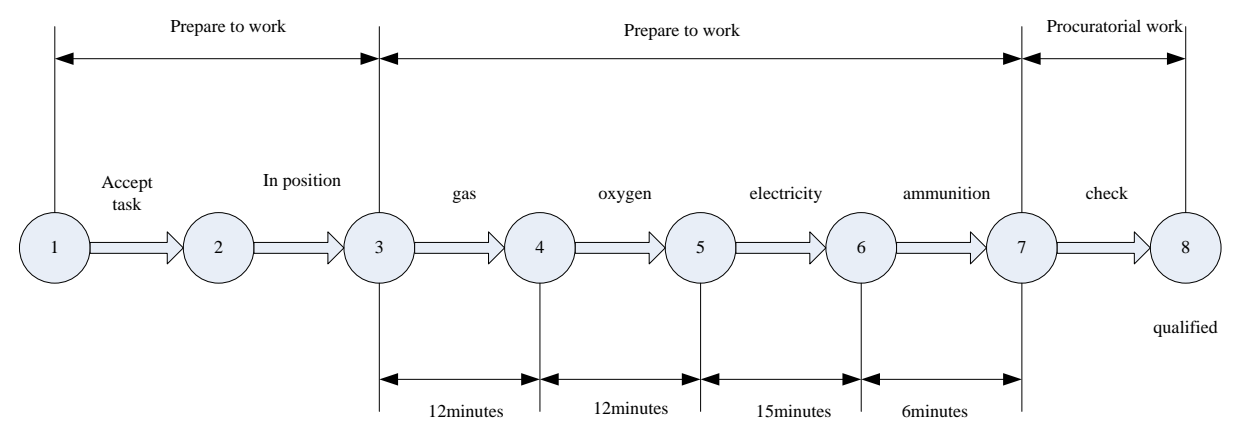

Figure 2. Security operation of single machine

Through the above analysis, we can work to guarantee the aircraft traditional approximate description for the open shop scheduling problem, the plane in the security environment as a "workshop" of work, the plane is equivalent to the workpieces, each kind of security equipment as processing machine, and the same kind of protection device can have multiple but not exceeding the amount of the aircraft. Since each aircraft's guarantee line is different and has no impact on each other. Process optimization is determined by the purpose of the executing order of tasks was different on each plane homework, can put all the aircraft in the shortest possible time to complete, reasonable make full use of resources.

The operational problems can be described as $\mathrm{n}$ planes being processed on $\mathrm{m}$ kinds of guarantee equipment. Each aircraft needs to carry out m-road protection procedure, and every guarantee operation has a definite working time. In a given time each safeguard device can only guarantee one plane at the same time, and each aircraft can only operate from one safeguard device. The order of processing of the aircraft on the same safeguard device is arbitrary, and the order of security operation of each aircraft is arbitrary. The interruption is not allowed after the job is started throughout the guarantee process. The purpose of ensuring the operation schedule is to determine the sequence of all aircraft and safeguard equipment in order to meet the above constraints, making 
the aircraft group to be the smallest.

The security issues of the machine should meet the following assumptions:

(1) if the job is started, the assignment will not be interrupted until the job is completed;

(2) there is no mutual restraint between different carrier-borne machines, and the operation content of all carrier-borne machines is independent of each other;

(3) there is no resource contention for hanging and inertial navigation, and the aircraft can be launched immediately after the aircraft is finished in parallel and serial.

(4) the same operating time of the same type of carrier is the same;

(5) a guarantee equipment resource does not allow for the protection of multiple carrier-borne machines at the same time;

(6) aircraft each operation can be performed in any security devices, but can only make a guarantee a homework, after one of the resources guarantee no longer needs the guarantee;

(7) each of the carrier-borne machines that arrive at the guarantee point shall have to perform all of the guaranteed work;

(8) allow the carrier aircraft to exist in operation time and ensure that the resources are idle;

(9) the guarantee work can be started at any time.

In preparation process efficiency, different models need to use different safeguard equipment, the amount and type is various, but the general and basic work mainly divides into four parts, namely "filling, add, add, hanging" : (1) "imitation" refers to all kinds of special gas filling, including oxygen, nitrogen and compressed air, etc., use of guarantee of equipment for all kinds of inflatable car, such as oxygen, nitrogen, models, etc. "Add" mainly refers to the addition of special oil, such as hydraulic oil, alcohol, various aviation lubricants, which need to be used to guarantee the vehicles for various small refueling vehicles. The "plus" mainly refers to the addition of fuel oil, the use of guarantee vehicles for refueling vehicles, or part ii and third-line guarantee for the use of refueling platform. "Hang" means the ordnance department is equipped with all kinds of weapons and ammunition, and the security equipment used is to hang a bullet train.

\section{Optimization of the Security Process of Multi-Machine}

Through computing has to finish four machine in 80 minutes before flight refueling, oxygen, nitrogen inflator, need all kinds of security equipment quantity respectively: two cars bowser, oxygen, nitrogen cars each one.

In order to optimize the guarantee process, the four aircraft are divided into two batches, T1 and $\mathrm{T} 2$ are the first batch, T3 and T4 are the second batch, and each batch is guaranteed according to the two-machine guarantee process. Adjust the T4 and T2 and T3 and T1 job order can shorten the waiting time, again will job sorting optimization, the optimized sequence of security vehicle configuration and security, synfuels fiasco complete security operation time of 25 points respectively, 27, 30, 30 points, and optimize the than before time shorten, needs the guarantee of resources also less, tanker truck down to two. The utilization rate of the guaranteed resources after optimization is shown in table 1 . The utilization ratio of oxygen vehicle and nitrogen vehicle was greatly improved compared with that before optimization. The utilization rate of oxygen vehicle increased by $27.4 \%$ and the utilization rate of nitrogen vehicle increased by $20.8 \%$.

Table 1 Statistics on the utilization rate of each guarantee equipment after optimization

\begin{tabular}{|c|c|c|c|}
\hline Ensure the vehicle & $\begin{array}{c}\text { Working hours } \\
\text { (minutes) }\end{array}$ & $\begin{array}{c}\text { Waiting time } \\
\text { (minutes) }\end{array}$ & utilization $(\%)$ \\
\hline tanker truck 1 & 30 & 0 & 100 \\
\hline tanker truck 2 & 30 & 0 & 100 \\
\hline Oxygen car & 24 & 3 & 88.9 \\
\hline Nitrogen car & 16 & 9 & 64.0 \\
\hline
\end{tabular}

In the case of meeting the guarantee time of 80 points, the order of the five-machine guarantee 
process can be obtained. The number of vehicles needed to ensure the safety of the vehicle is two, the oxygen vehicle and the nitrogen vehicle each. The plane of security can be divided into two batches, each batch according to the 3 in 1 support process support, by adjusting oxygen filling sequence of T2 can shorten the waiting time, will arrange homework after T3 and T2 will optimize again job sorting, complete synfuels fiasco oxygen filling the job will shorten the six points. The time required for each machine to complete the work is $31,35,36,42$ and 45 , which can meet the need of guarantee. After optimization, the utilization statistics of each safeguard equipment are shown in table 2. After optimization, the utilization rate is still higher than before optimization, and there is less demand for the quantity of the equipment, and only 2 vehicles are needed for the refueling vehicles.

Table 2 The utilization statistics of each safeguard equipment after optimization

\begin{tabular}{|c|c|c|c|}
\hline Ensure the vehicle & $\begin{array}{c}\text { Working hours } \\
\text { (minutes) }\end{array}$ & $\begin{array}{c}\text { Waiting time } \\
\text { (minutes) }\end{array}$ & utilization $(\%)$ \\
\hline tanker truck 1 & 15 & 0 & 100 \\
\hline tanker truck 2 & 15 & 0 & 100 \\
\hline Oxygen car & 30 & 12 & 71.4 \\
\hline Nitrogen car & 20 & 15 & 57.1 \\
\hline
\end{tabular}

In the same way, you can get the order of the six - machine guarantee process, and the required number of vehicles will be two, oxygen and nitrogen vehicles respectively. To ensure the aircraft is divided into two batches, each batch according to the compressors to ensure process, all the plane completed homework takes 45 minutes, T1 plane complete security work need 31 minutes, T2 aircraft through security work to 35 minutes, T2 plane complete security work need 36 minutes, TS plane complete security work need 36 minutes, finally T3, T6 aircraft through security work to 45 minutes, can satisfy the guarantee time of 80 minutes of preparation conditions, and all the free time minimum security resources, in the six machine gas, oxygen, nitrogen filled the sorting of these three work assignments for optimal.

Optimized the safeguard equipment utilization statistics are shown in table 3 below, optimized the security vehicle utilization is still greater than the former, and the demand for security equipment is less, the optimized to meet the need for the task.

Table 3 The utilization statistics of each safeguard equipment after optimization

\begin{tabular}{|c|c|c|c|}
\hline Ensure the vehicle & $\begin{array}{c}\text { Working hours } \\
\text { (minutes) }\end{array}$ & $\begin{array}{c}\text { Waiting time } \\
\text { (minutes) }\end{array}$ & utilization $(\%)$ \\
\hline tanker truck 1 & 45 & 0 & 100 \\
\hline tanker truck 2 & 30 & 0 & 100 \\
\hline Oxygen car & 36 & 12 & 75.0 \\
\hline Nitrogen car & 24 & 15 & 61.5 \\
\hline
\end{tabular}

In the same way, you can get the order of the nine machine guarantee procedures, and the required number of vehicles will be 3 cars, oxygen trucks and nitrogen vehicles respectively. To ensure the aircraft is divided into three groups, each group according to the compressors to ensure process guarantee, all aircraft to complete basic safeguard work time is 54 points, 4 points within the prescribed time, but considering the operation cost and resource use efficiency, this kind of resource allocation and scheduling is still for the most optimal selection.

Because of the limited fuel vehicles, only 6 aircraft are equipped, so it is not guaranteed that each aircraft will be equipped with a refueling vehicle according to the traditional guarantee mode. It takes 73 minutes to complete all 9 machines, much more than the required pre-flight guarantee time. The utilization statistics of the security equipment after optimization are shown in table 4. 
Table 4 The utilization statistics of each guarantee equipment after optimization

\begin{tabular}{|c|c|c|c|}
\hline Ensure the vehicle & $\begin{array}{c}\text { Working hours } \\
\text { (minutes) }\end{array}$ & $\begin{array}{c}\text { Waiting time } \\
\text { (minutes) }\end{array}$ & utilization (\%) \\
\hline tanker truck 1 & 45 & 0 & 100 \\
\hline tanker truck 2 & 45 & 0 & 100 \\
\hline tanker truck 3 & 45 & 0 & 100 \\
\hline Oxygen car & 54 & 0 & 100 \\
\hline Nitrogen car & 36 & 4 & 90 \\
\hline
\end{tabular}

After optimization, to increase the utilization rate of oxygen and nitrogen models, a tanker truck utilization rate of $100 \%$ of the nitrogen and oxygen car car utilization rate has reached $90 \%$, at the same time ensuring the whole process of tanker truck need less, only need three, can achieve the purpose of saving resources and efficient use of equipment.

\section{Equipment Quantity Allocation and Operation Sequence Optimization Analysis}

By this method can guarantee different number of plane process of sorting, under the same allocation must be prepared to safeguard equipment efficiency, through the optimization of the process for security, improve the efficiency of the safeguard equipment, thus the maximum extent, shorten the preparation time efficiency. Guarantee time needed for different number of plane as shown in table 6, the data can be obtained from the table, the increase in the number of security in accordance with the traditional time to finish the three basic safeguard security operation mode gradually increase, more than six machine has serious impact on the efficiency of the preparation efficiency. These three work is parallel with the rest of the security work, to guarantee the highest efficiency, guarantee the shortest time should try to make all these three work complete time with the rest of the professional security operation time, the rest time to finish the preparation efficiency professional for 44 points, so the complete gas, oxygen, nitrogen filled into the best time to 44 .

During the actual flight implementation, the maximum number of the single batch is six, which is restricted by the runway, the power supply air conditioning and other safeguard equipment, as well as the combat mode. Can be seen from the table in the optimized completion to six single machine security operation for an average of 34.2 points, can satisfy the actual needs, and optimize the need for an average of 40.8 points before, can also meet the needs of training and battle.

With the development of security technology, security equipment renewal and the transformation of the combat mission, single batch of vehicles has become inevitable, so the optimization of equipment configuration and security model is under the existing in the current equipment important means to improve the quality of preparation. But as the number of aircraft single batch of security gradually increased, the traditional security mode when nine machine, time of 73 points, the serious influence the flight, can't satisfy the guarantee of mission, and the guarantee of the optimized model is greatly reduces the operation time, guarantee the plane more than nine machine, oxygen, nitrogen filled security car will increase each one, refueling equipment also increases accordingly, preparation time will also keep in 45 minutes. Therefore, optimized configuration and guarantee order are more suitable for the preparation of multiple aircraft.

\section{Concluding Remarks}

Likewise, equipment utilization and optimization of the important indicators, improve the utilization ratio can be taken to reduce the number of security resources, cost savings, effectively reduce equipment idle time, complete security tasks more quickly. Due to the highest priority of the refueling operation, the optimized work is the first to be done before and after the optimization, with the highest utilization rate, so it is not considered the optimization of the fuel efficiency. With the increase of the increase of the number of aircraft, the average utilization rate is 47.0 percent and the nitrogen vehicle is 37.4 percent. 
As safeguard the plane also rises with an increase in the number of utilization, from security to six single machine overall stability between $50 \%$ and $80 \%$, the average utilization rate is $63.4 \%$ nitrogen oxygen car is $63.4 \%$, had increased by $16.4 \%$ and $4.4 \%$ respectively, the basic achieve the goal of the optimization.

\section{References}

[1] Sriram Venkataramanan, Atilla Dogan.A Multi-UAV Simulation for Formation Reconfiguration[R]. AIAA2004-4800. Providence, Rhode Island:AIAA Modeling and Simulation Technologies Conference and Exhibit,2003

[2] Jerome Azarewicz, Glenn Fala. Template-based Multi-agent Plan Recognition for Tactical Situation Assessment [A]. In:Proceedings of 5th conference on Artificial Intelligence Applications[C], March 1989:247-254

[3] M.Valenti, T.Schouwenaars, Y.Kuwata, E.Feron and J. How Implementation of a Manned Vehicle-UAV Mission System[C].AAIA Guidance, Navigation and control Conference, Providence, RI, August 2004,AIAA2004 